\title{
Carbonyl Iron Foam Surfaces Modified with Poly (L-Lysine) As Smart Surface for Bone Implant
}

\author{
Jan Macko', Carlos Echeverría-Arrondo², Natália Podrojková ${ }^{1}$, Yazmin Angelina Brito \\ Barrera $^{3}$, Husnia Kindi ${ }^{3}$, Katarína Sisáková1 ${ }^{1}$ Radka Gorejová ${ }^{1}$, Rastislav Jendželovsky ${ }^{4}$, \\ Viktória Bul'ková ${ }^{4}$, Iván Mora-Seró ${ }^{2}$, Victor Sans ${ }^{2}$, Renáta Oriňaková ${ }^{1}$ and Thomas Groth ${ }^{3}$ and \\ Andrej Oriňak ${ }^{1 *}$ \\ ${ }^{1}$ Institute of Chemistry, Department of Physical Chemistry, Pavol Jozef Šafárik University in Košice, Moyzesova 11, Slovak Republic
} ${ }^{2}$ Institute of Advanced Materials (INAM), University Jaume I, Spain

${ }^{3}$ Department Biomedical Materials, Interdisciplinary Center of Materials Science, Institut für Pharmazie, Martin-Luther-Universität Halle-Wittenberg, Heinrich-Damerow-Str. 4, Germany

${ }^{4}$ Institute of Biology and Ecology, Department of Cellular Biology, Faculty of Science, Pavol Jozef Šafárik University in Košice, Šrobárova 2, Slovak Republic

*Corresponding author: Andrej Oriňak, Institute of Chemistry, Department of Physical Chemistry, Pavol Jozef Šafárik University in Košice, Moyzesova 11, Slovak republic.

To Cite This Article: Jan Macko, Carlos Echeverría-Arrondo, Natália Podrojková, Yazmin Angelina Brito Barrera, Andrej Oriňak, et al., Carbonyl Iron Foam Surfaces Modified with Poly (L-Lysine) As Smart Surface for Bone Implant. Am J Biomed Sci \& Res. 2021 - 14(3). AJBSR.MS.ID.001985. DOI: 10.34297/AJBSR.2021.14.001985.

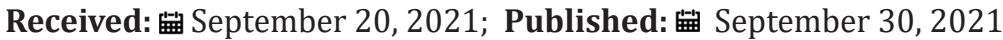

\begin{abstract}
This article presents the surface modification of iron Fe (110) surfaces with Poly-L-Lysine (PLL) with the aim of preparing carbonyl iron bone implants which are less corrosive and more compatible with fibroblast cells. The cytocompatibility of modified surfaces with commercially available $\alpha$-PLL and electrodeposited $\varepsilon$-PLL was compared by combination of DFT computational simulations with experimental electrochemical and cell adhesion studies to obtain "smart" surface application. Experimental study of fibroblasts adhesion showed better viability of cells on $\varepsilon$-PLL than on $\alpha$-PLL after modification of Fe surfaces as "smart" surfaces to obtain a different hydrophobicity. The porosity of Fe (110) prevented direct measurements of contact angle and therefore surface hydrophobicity was simulated with calculation of adsorption energies for Fe with both $\alpha$-/ $\varepsilon$ PLL structures. This technique was also employed to calculate the interaction of O-H bonds at the surface. The corrosion potential of Fe (110) with superficially modified $\varepsilon$-PLL was shifted by $0.088 \mathrm{~V}$ compared to the bare iron surface, thus indicating a stronger resistance to corrosion. The results suggest that modification of Fe surface with $\varepsilon$-PLL has a more pronounced effect on cellular growth on this implant and that the slightly hydrophobic character of $\varepsilon$-PLL leads to better cell adhesion ability.
\end{abstract}

Keywords: $\alpha$-/ $\varepsilon$-Poly-L-Lysine; Carbonyl Iron Foam; Surface Modification; Adhesion Modulation; DFT Models; Bone Implant; Biocompatible; Biomolecules; Carboxyl; Biosynthesized; Amino; Endotoxin

\section{Introduction}

Poly-L-Lysine (PLL) represents a biocompatible polymer which as cationic structure becomes an extraordinarily effective molecular coating for the adhesion of negatively charged biomolecules, generally DNAs [1]. Biosynthesized ( $\varepsilon$-PLL) carries R-amino groups on the side chains which can react with the residual amino and carboxyl groups resulting in formation of an anion-cationic pair called the zwitterion. The so formed naturally occuring zwitterion displays antifouling activity when molecules of water interact with the surface of the material, which may suppress the adhesion of the bacteria [2]. As a result of such polycationic nature associated with biodegradability and chirality the homopolymer of the amino acid L-lysine has impressive properties which enables PLL modified layers to be studied in various biochemical applications including sterilization, antisepsis, gene transfection and endotoxin removal [3]. 
PLL-based nanomaterials are also being studied to increase biocompatibility, promote cell adhesion, and enhance drug distribution [4] and linear and dendritic PLL structures have been employed, for example, in tissue engineering, or as prophylactic for viral infections [1] Electrostatic self-assembly nanotechnology constitutes a promising way of generating thin layers with highly controlled molecular architectures [5,6]. Another interesting possibility of application of PLL is adsorption on different materials for surface modification and as a method of layer-by-layer fabrication [7]. In general, the appropriate functionalization of metal surfaces with biomolecules that keep their conformation and activity remains a challenge [8]. In the case of iron bone implant surface modification with PLL a corrosion plays an important role. Application of corrosion inhibitors for the protection of steel is also used and investigated by many authors [9]. The highest protection against metal corrosion is provided by organic molecules that contain heteroatoms such as oxygen, sulfide, and nitrogen [10].

Researchers show that most organic inhibitors work by a mechanism of adsorption on the metal surface. This mechanism involves blocking active sites on the iron surface due to the adsorption of inhibitor molecules. Since the metal surface is covered primarily by adsorbed $\mathrm{Cl}$ - ions, amino acid cations are able to electrostatically adsorb on the surface [11]. The lysine adsorbs onto the metallic surfaces by interaction with the $\varepsilon$-amino group [2]. The conformational state of PLL at the molecular level is determined by the degree of electrostatic repulsion between its side chains and hydrogen bonds. The presence of PLL provides superior hydrophilicity and roughness against titanium disc implant surface and safely promotes osteoblast calcium deposition [12]. Surface hydrophilicity plays a significant role in the interaction of cells with the surface. If the surface is only slightly hydrophilic, it is difficult for the cells to adhere. Adhesion and cell spreading cohere with the dispersion and polar components of the energy of the surface [13].

Highly tuned polymer brushes can be used to overcome any limitation with robust coatings used to adjust a great diverse of biological substrates. To enhance integration of implant-bone, titanium substrate can be coated with a SAM-oligoethylene glycol brushes-synthetized by surface-initiated atom transfer radical polymerization that can effectively promote bone cell differentiation in vitro and implant integration [14]. Computational modelling with the Quantum Espresso code [15] (based on plane waves, and with Projector Augmented-Wave (PAW) pseudopotentials generated in the GGA approximation for the exchange-correlation functional), is used to apprehend the adsorbate-surface interactions. Atomic and molecular adsorption on Fe (110) is well documented [15,16]. Since the measurement of contact angle and wettability can be difficult and sensitive to ambient conditions it is important to include computational modelling. In addition to the experimental research, the theoretical study of the hydrophobicity of $\mathrm{CeO}_{2}$ surfaces is necessary for a better understanding of surface behavior. Fronzi et al. studied the hydrophobicity of Cerium (IV) oxide by DFT method [16].

They calculated water double layer adsorption energies at $\mathrm{CeO}_{2}$ (100), (110) and (111) surfaces, contact angle and surface hydrophobicity with use of computational modeling. Nowadays, the computational research is also focused on the calculation of the hydrophobicity at the surface of proteins [17], for instance the adsorption of chiral L-lysine on Cu (110) surfaces [18] and L-lysine general adsorption geometry [19]. Computational modelling has provided an insight into the adsorbate-surface interactions at the molecular level. In the present study, we have examined modification of iron foam material prepared by the sintering of carbonyl iron powder with different forms of PLL for adhesion of fibroblast cells. Additionally, the adsorption process of the PLL from the acidic solution onto the iron surface was provided by DFT calculations, in which adsorption geometries of PLL on the Fe (110) slab were considered. Our computational results indicate that PLL confers a higher resistance to corrosion and insights in how the morphology of the PLL layer and its hydrophobicity plays a role in the cytocompatibility of the "smart" surface with potential application as bone implant.

\section{Results and Discussion}

\section{Results of DFT studies of $\varepsilon$-PLL}

Firstly, the water adsorption on the iron surface (110) was modeled with initial proposals for the water coating layer displayed in Figure 1 and the $\alpha-/ \varepsilon$-PLL layer presented on Figure 2. Then, DFT simulations were investigated to model the adsorption of the $\alpha-/ \varepsilon$ PLL on the Fe (110) surface to find how the molecules bind onto the iron surface and to study the surface hydrophobicity. The geometry of $\alpha-/ \varepsilon$-poly-L-lysine is displayed on Figure 2. The differences in structure and geometry can be appreciated from their respective side views. Adsorption energies calculated are given in Table 1. The lowest adsorption energy was found for $\alpha$-PLL and the contact angle indicates that $\alpha$-PLL is a hydrophilic surface. Oppositely, the value of adsorption energy of $\varepsilon$-PLL was calculated as high as $-0.39 \mathrm{eV}$. Furthermore, the calculated contact angle indicated that this surface was slightly hydrophobic. These results were obtained in the good relations with calculations done in reference [18]. 

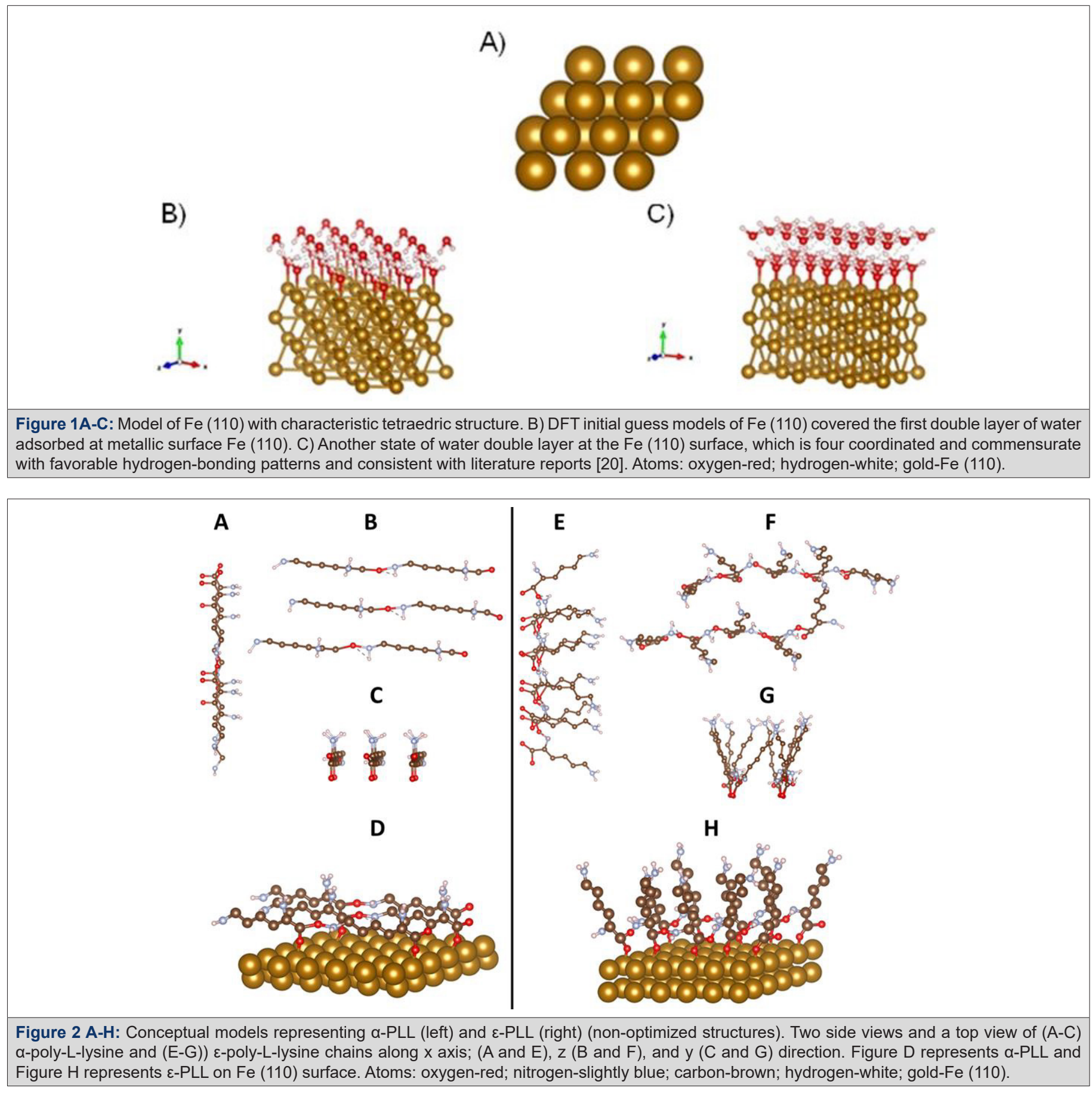

Table 1: Calculated adsorption energies of different PLL bond on Fe (110).

\begin{tabular}{|c|c|c|c|}
\hline Entry & Surface & EAdh/[eV] & Contact angle $\boldsymbol{\theta}$ \\
\hline 1 & Ead (H20) at Fe (110): (per water molecule) & -0.42 & $67.80^{\circ}$ \\
\hline 2 & E ad (H20) at Fe (110)-ع- PLL) & -0.39 & $73.01^{\circ}$ \\
\hline 3 & E ad (H20) at Fe (110)- $\alpha$-PLL) & -0.57 & $49.96^{\circ}$ \\
\hline
\end{tabular}

It is worth mentioning that the porosity of the material made very challenging the use of experimental methods to determine the contact angle, and therefore the DFT method here presented has proven to be effective to characterize either surface hydrophobicity or hydrophilicity. Typically, nearly every animal cell favors moderately hydrophilic surfaces in terms of adhesion and growth. The hydrophobicity over the molecular surface of $\alpha$-PLL is caused by the surface which is linearly bound through oxygen atoms as 
shown on Figure 2D where a simulated structure of the molecule is shown. This surface is more hydrophilic as in the case of the surface of $\varepsilon$-PLL (Figure 2E, H) which is weakly hydrophobic due to elevated molecular brushes of PLL. It is known that cell adhesion and spreading is associated with the polar and dispersion components of free surface energy. Harnett et. al. investigated the influence of surface energy components on cell spreading and cell division rates of a transformed line of mouse lung fibroblasts on various polymers in the presence of serum proteins [13]. The ideal surface contact angle where the strongest cell attachment is observed varies from $5^{\circ}$ to $40^{\circ}[20]$.

(Figure 1A) Model of Fe (110) with characteristic tetraedric structure B DFT initial guess models of Fe (110) covered the first double layer of water adsorbed at metallic surface Fe (110) C) Another state of water double layer at the Fe (110) surface, which is four coordinated and commensurate with favorable hydrogenbonding patterns and consistent with literature reports [20]. Atoms: oxygen-red; hydrogen-white; gold-Fe (110). Figure 2 Conceptual models representing $\alpha$-PLL (left) and $\varepsilon$-PLL (right) (non-optimized structures). Two side views and a top view of (A-C) $\alpha$-poly-L-lysine and (E-G)) $\varepsilon$-poly-L-lysine chains along $x$ axis; ( $A$ and $E$ ), z (B and F), and y ( $C$ and $G$ ) direction. Figure D represents $\alpha$-PLL and Figure $H$ represents $\varepsilon$-PLL on Fe (110) surface. Atoms: oxygen-red; nitrogen- slightly blue; carbon-brown; hydrogen-white; gold-Fe (110). Table 1 Calculated adsorption energies of different PLL bond on Fe (110).

Figure 3 DFT simulated models of $\alpha-/ \varepsilon$-PLL. A-surface of Fe (110); B-surface of Fe (110) covered with double water layer for calculating hydrophobicity; C- $\alpha$-PLL bond on Fe (110); D-optimized structure of $\alpha$-PLL; E-optimized structure of $\varepsilon$-PLL bond on $\mathrm{Fe}$ (110); F-optimized structure of $\varepsilon$-PLL bond through water double layer; Atoms: oxygen-red; nitrogen-slightly blue; carbon-brown; hydrogen-white; gold-Fe (110). The understanding of the basis of the various levels of both hydrophobicity or hydrophilicity on Fe (110) $\alpha-/ \varepsilon$-PLL surface coatings demands an analysis of the interactions between hydrogen atoms of amino groups of PLL surface and the oxygen atoms and calculations of crystal overlap population. H-O bonds with higher intensity and deeper in the valence band indicated stronger hydrophilic Fe (110) PLL-water interactions. Amino groups have also affected surface hydrophilicity of PLL by the formation of hydrogen bridges with oxygen atoms of carboxylic groups as it is clear from shown in detailed Figure 4. The angle between hydrogen of amino group and oxygen atom carboxylic group is shortened, which increases the hydrophilicity of the system. The terminal amino group seems to decrease the hydrophobicity of the surface about $60 \%$ according to the results of the calculations.

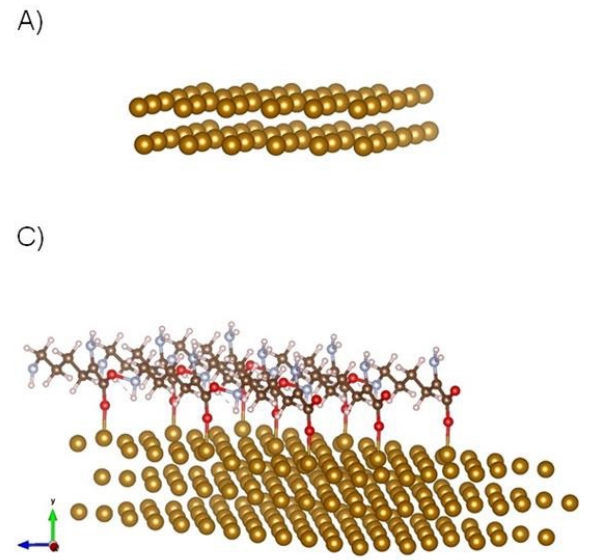

E)

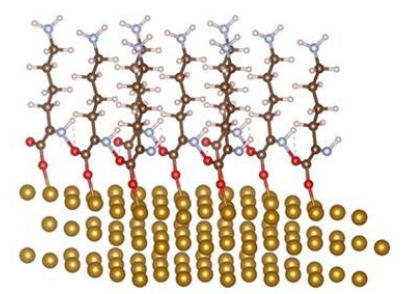

B)

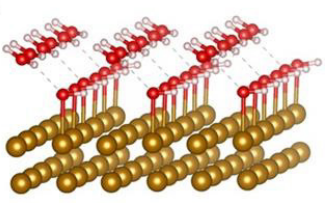

D)

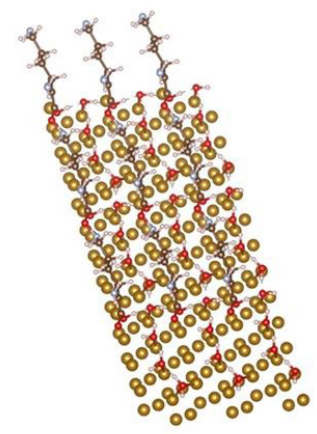

F)

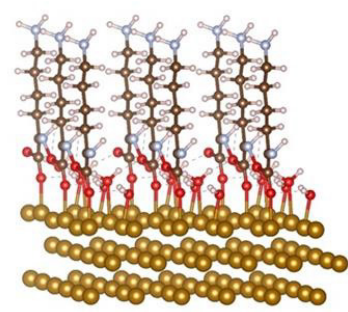

Figure 3A-F: DFT simulated models of $\alpha-/ \varepsilon-P L L$. A) Surface of Fe (110); B) Surface of Fe (110) covered with double water layer for calculating

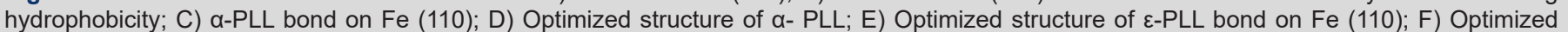
structure of $\varepsilon$-PLL bond through water double layer; Atoms: oxygen-red; nitrogen-slightly blue; carbon-brown; hydrogen-white; gold-Fe (110). 
Figure 4 Comparison of bond angles between $\alpha$ - and $\varepsilon$-PLL on Fe (110) surface A) Structure of $\varepsilon$-PLL-Specific framework structure of angles and bonds between adjacent peptide bonded $\varepsilon$-PLL molecules. It is clearly seen that hydrogen forms bridges with oxygen with their corresponding bond and angle is shortened to $103.3^{\circ} \mathrm{B}$ )-Specific framework structure of angles and bonds between adjacent peptide bonded $\alpha$-PLL molecules. The angle between $\mathrm{O}$ and $\mathrm{H}$ is greater and it is clearly seen that hydrogen and oxygen interaction is weaker. The greatest change of angle was observed between $\mathrm{C}$ and $\mathrm{O}$ atoms. Atoms: oxygen-red; nitrogen- light blue; carbon-brown; hydrogen-white; gold-Fe (110). Adhesion and viability of fibroblast at L-lysine and different PLL modified Fe (110) surface PLL is a positively charged polymer containing a broad number of amino groups (a model of PLL can be found in the Figure SX in the SI) which are predominantly unprotonated beneath physiologic conditions, with ability to increase wettability of the materials. According to earlier studies the ideal surface contact angle applicable for cell cultivation varies from $5^{\circ}$ to $40^{\circ}$ which induces enhanced cell adhesion and growth on desired surfaces with such hydrophilic nature [20].
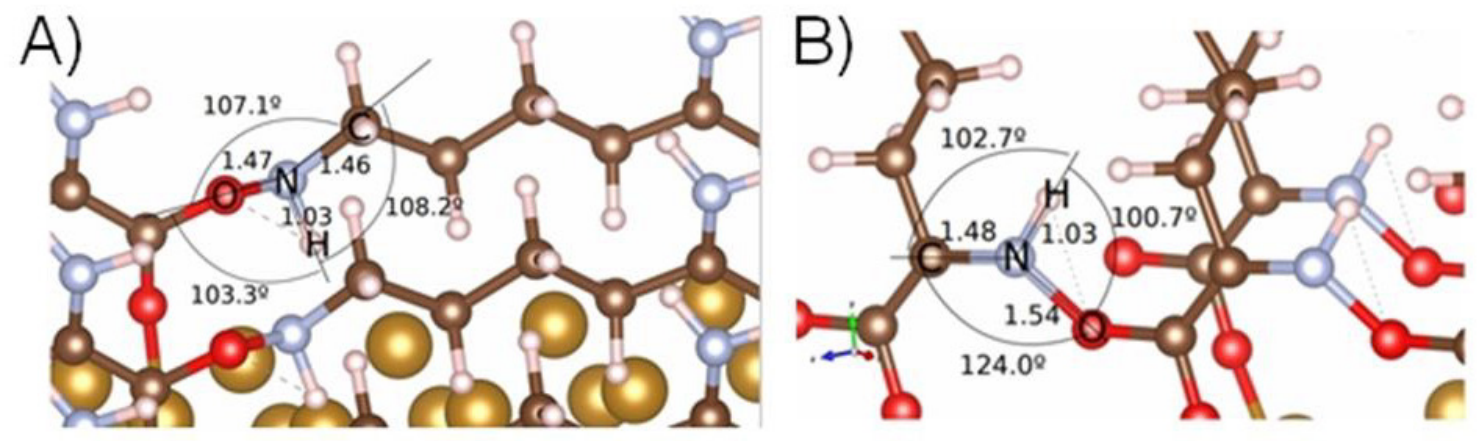

Figure 4 A,B: Comparison of bond angles between $\alpha$ - and $\varepsilon$-PLL on Fe (110) surface. A) Structure of $\varepsilon$-PLL-Specific framework structure of angles and bonds between adjacent peptide bonded $\varepsilon$-PLL molecules. It is clearly seen that hydrogen forms bridges with oxygen with their corresponding bond and angle is shortened to $103.3^{\circ}$. B) Specific framework structure of angles and bonds between adjacent peptide bonded $\alpha$-PLL molecules. The angle between $\mathrm{O}$ and $\mathrm{H}$ is greater and it is clearly seen that hydrogen and oxygen interaction is weaker. The greatest change of angle was observed between $\mathrm{C}$ and $\mathrm{O}$ atoms. Atoms: oxygen-red; nitrogen-light blue; carbon-brown; hydrogen-white; gold-Fe (110).
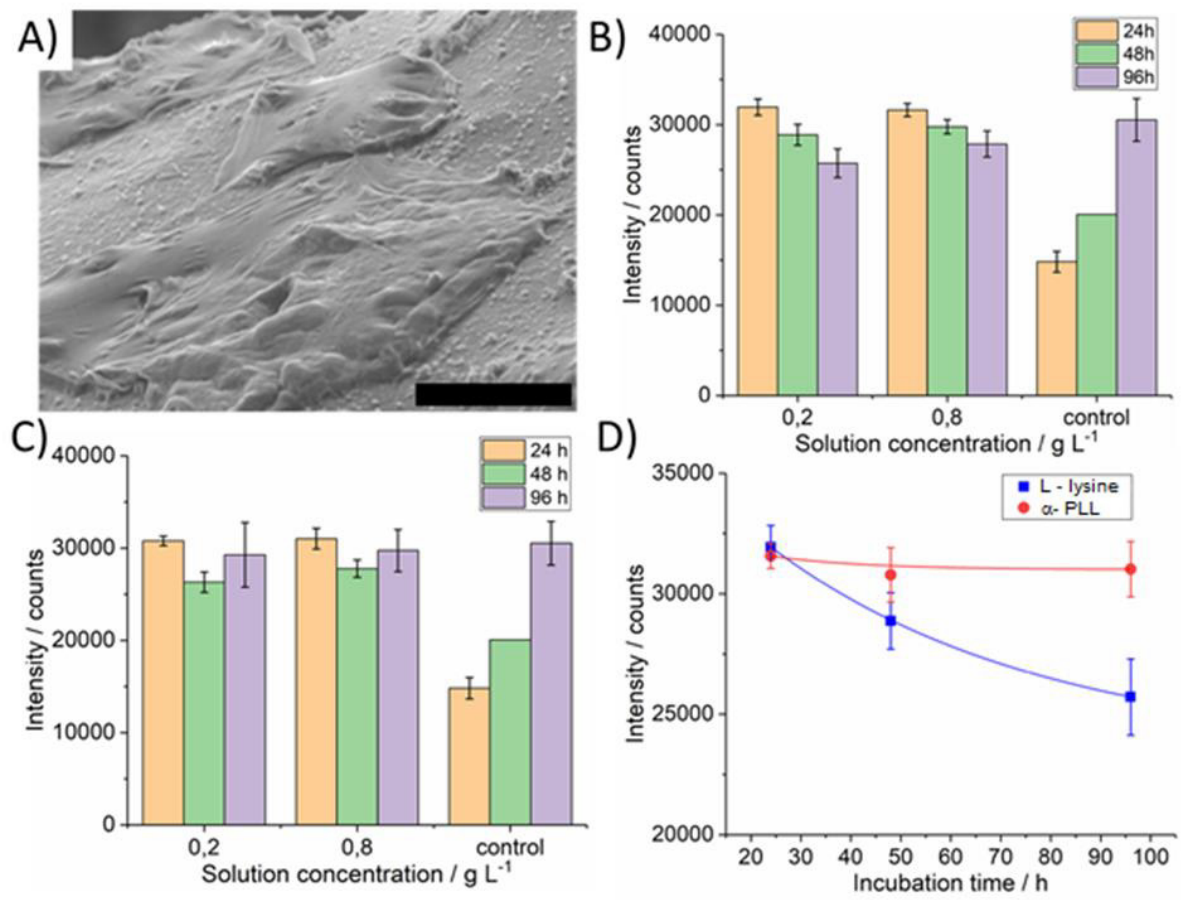

Figure 5A-D: Micrograph of Fe (110) surface with growth fibroblast cells. A) Cell viability evaluation using Deep Blue cell viability kit on iron cellular material surface functionalized by L-lysine. B) $\alpha$ - poly(L-lysine)

molecules. C) Relationship of incubation time on intensity of counts. D) Measurements were performed after 24 (pink bar), 48 (green bar) and 96 (blue bar) hours after cell seeding. Size of the scale bar $20 \mu \mathrm{m}$. 
PLL is frequently used to modify surfaces to promote cell adhesion since it reacts strongly with negatively charged molecules and cells. PLL surface modification is simple and convenient [21]. Hydrophilicity plays an important role in cell substrate interaction. Likibi et al. showed that an increasing number of adhered cells with increasing polypeptide coating [22]. PLL modified surface and cell interaction is mainly caused due to the positive charge of PLL and negative charge of the cell membrane [22]. Results of viability fibroblast on Fe (110) surface modified with $\alpha$-poly-L-lysine. Cells were found to adhere adequately on $\alpha$-PLL surfaces, as evidenced by SEM images (Figure 5A). It was found that fibroblasts cells were adhered with the same intensity as with L-lysine in the early stages of cellular growth. Polypeptide-coated samples were noncytotoxic compared with controls (Figure 5B-C). Fibroblast stopped growth after 24 hours of cultivation stopped growth. But after 48 hours it began to grow again.

Figure 5 Micrograph of Fe (110) surface with growth fibroblast cells-A. Cell viability evaluation using Deep Blue cell viability kit on iron cellular material surface functionalized by L-lysine-B and $\alpha$ - poly (L-lysine) molecules-C. Relationship of incubation time on intensity of counts-D. Measurements were performed after 24 (pink bar), 48 (green bar) and 96 (blue bar) hours after cell seeding. Size of the scale bar $20 \mu \mathrm{m}$. It is clearly seen; cells have a high level of viability after 24 hours after seeding. The relative fluorescence intensity is little more than two times higher compared to control
(Figure 5B-C). After 48 hours the relative fluorescence intensity is a little lower, but the number of viable cells there was still significant. At the end of the experiment, after 96 hours the relative fluorescence intensity of control samples was little higher than the intensity of examined samples thus all. When compared to Figure 5B and Figure $5 \mathrm{C}$ a significant difference is seen. Cell viability after 96 hours in case of $\alpha$-PLL functionalized surface is slightly increased compared to that on surface functionalized with L-lysine functionalized ones. Hence, it can be concluded that $\alpha$-PLL functionalized iron cellular materials have better biocompatibility and lower cytotoxicity. Results of viability fibroblast on Fe (110) surface modified with $\varepsilon$-poly-L-lysine. A micrograph of $\varepsilon$-PLL modified carbonyl Fe (110) surface from different places is shown on figure Figure 6.

(Figure 6A) Various SEM images of bone implant surface continuously covered by white of $\varepsilon$-poly-L-lysine layer formed by electro polymerization. Look from different places. Magnification A 100x, B 100x, C 500x, D 1000x. Cell viability evaluation using Deep Blue cell viability kit on iron cellular material surface functionalized by electrochemically polymerized $\varepsilon$-poly-L-lysine molecules. Measurements were performed after 24 (blue, brown bar) and 48 (deep orange, green bar) hours after cell seeding. Potentiodynamic polarization curves of untreated and $\varepsilon$-PLL layer modified iron cellular materials in $1 \mathrm{X}$ PBS solution at $25^{\circ} \mathrm{C}$; Measurements of the Open Circuit Potential (OCP). Size of the scale bars: $200 \mu \mathrm{m}$ (blue), $100 \mu \mathrm{m}$ (orange) and $20 \mu \mathrm{m}$ (red).

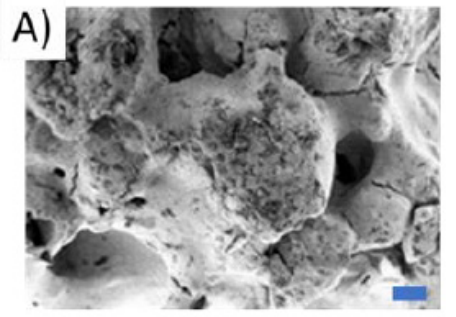

B)

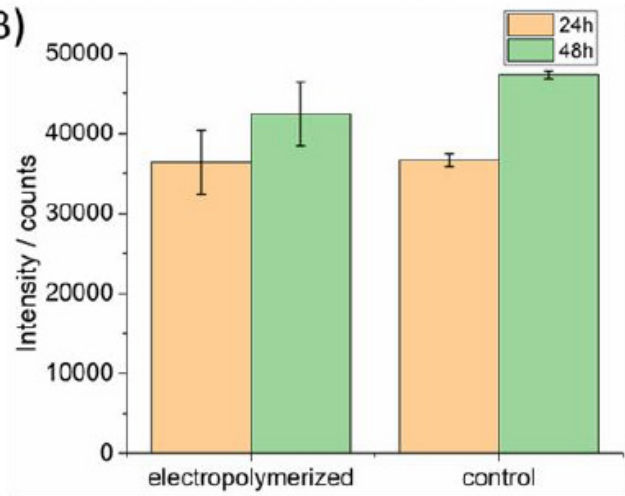

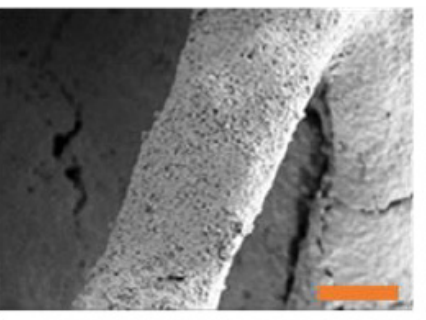

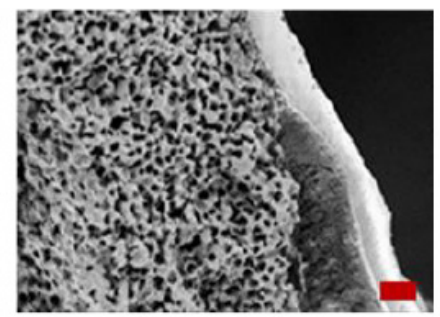

C)

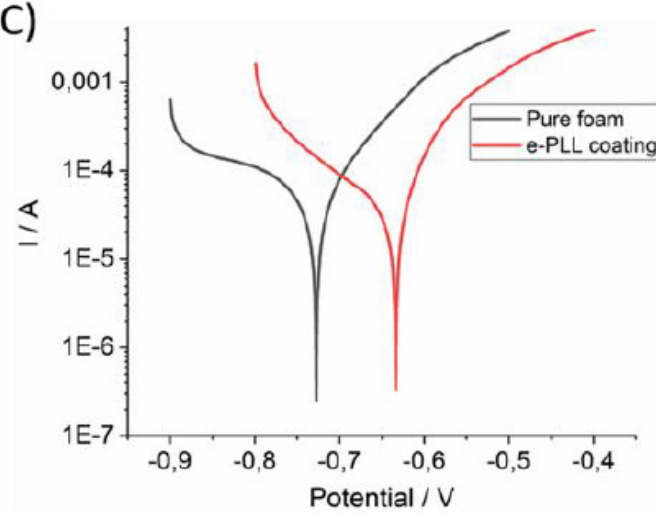

Figure 6A-C: Various SEM images of bone implant surface continuously covered by white of $\varepsilon$-poly-L-lysine layer formed by electro polymerization Look from different places. Magnification A 100x, B 100x, C 500x, D 1000x. Cell viability evaluation using Deep Blue cell viability kit on iron cellular material surface functionalized by electrochemically polymerized $\varepsilon$-poly-L-lysine molecules. Measurements were performed after 24 (blue, brown bar) and 48 (deep orange, green bar) hours after cell seeding. Potentiodynamic polarization curves of untreated and $\varepsilon$-PLL layer modified iron cellular materials in $1 \mathrm{X} \mathrm{PBS}$ solution at $25^{\circ} \mathrm{C}$; Measurements of the Open Circuit Potential (OCP). Size of the scale bars: $200 \mu \mathrm{m}$ (blue), $100 \mu \mathrm{m}$ (orange) and $20 \mu \mathrm{m}$ (red). 
When compared to the previous cases results, $\varepsilon$-PLL (Figure 5), Figure 6 showed the increase of fibroblast cell viability with cell cultivation duration (Figure 6) compared to previous cases. The presented electrochemically polymerized $\varepsilon$-PLL coating can be considered as biocompatible and non-cytotoxic. One of the possible reasons of higher cell viability is fact that Figure 5 shows evaluation of cell viability of $\alpha$-PLL modified iron cellular material and Figure 6 shows iron cellular material modified by $\varepsilon$-PLL, which is considered as nontoxic [23], when compared to its $\alpha$ form [24]. The relation between wettability and cell adhesion has been proven in many studies. Viability on to the $\alpha$-PLL in case of both concentrations stabilized at intensity of 28000.

Cell viability of the electrochemically polymerized $\varepsilon$-PLL iron cellular material stabilized at significantly higher value of about 35000. Polyethylene surfaces with wettability gradient from $96^{\circ}$ to $43^{\circ}$ were created using corona discharges [18]. There was also investigated interaction with various cell lines and fetal bovine serum [17]. Cell spreading and growth was examined predominantly on the surfaces with moderate hydrophilicity. Maximum was observed at $57^{\circ}$ for every cell type tested. This observation agrees with performed DFT Quantum ESPRESSO PLL simulation (see figures 2-3). The level of cell adhesion may be influenced by the thickness of the coating layer. In reference [24] it is documented that a higher number of cells adhered on one layer of PLL coated samples when compared to the control on the one bilayer of the PLL/PLGA-coated samples. The possible reason for this is the positive charge of PLL while most cell surfaces are negatively charged, which results in more cells attaching to the PLL coated surface.

\section{Corrosion Analysis of Fe (110) Modified With $\varepsilon$-PLL}

The $\varepsilon$-PLL modified samples revealed a higher corrosion potential $(-0.634 \mathrm{~V})$ compared to the potential of untreated iron cellular material $(-0.722 \mathrm{~V})$, showing that the iron cellular material modified by electropolymerized PLL layer coating increased the anticorrosive characteristics of PLL modified iron cellular materials. Such corrosion rates were established at values: $0.14 \mathrm{~mm} /$ year for carbonyl ICM and $0.07 \mathrm{~mm} /$ year for $\varepsilon$-PLL coated carbonyl iron. Total ECorr difference was about $0.088 \mathrm{~V}$. In general, the corrosion potential ECorr specifies the corrosion difficulty criterion thermodynamically. With increasing corrosion potential, the anticorrosive characteristics are improving.

\section{Suplement}

\section{Materials and methods}

Chemicals: L-lysine hydrochloride was purchased from Glentham Life Sciences Ltd. UK, $\alpha$-poly-L-lysine hydrobromide was purchased from Alamanda polymers Inc., USA, Deep Blue Cell Viability Kit BIOZOL, Germany, iron CC d 50 was purchased from BASF, Germany, hydrochloric acid, DMEM, PBS, Trypsine, FBS, PenStrep antibiotics were purchased from Sigma Aldrich, USA Figure 7 Schemes of different structures of PLL: a $\alpha$-PLL b, $\varepsilon$-PLL c, PLL dendrigraft G2 [25] is a combination of poly-L-lysines with an average of 48 Lys residues.

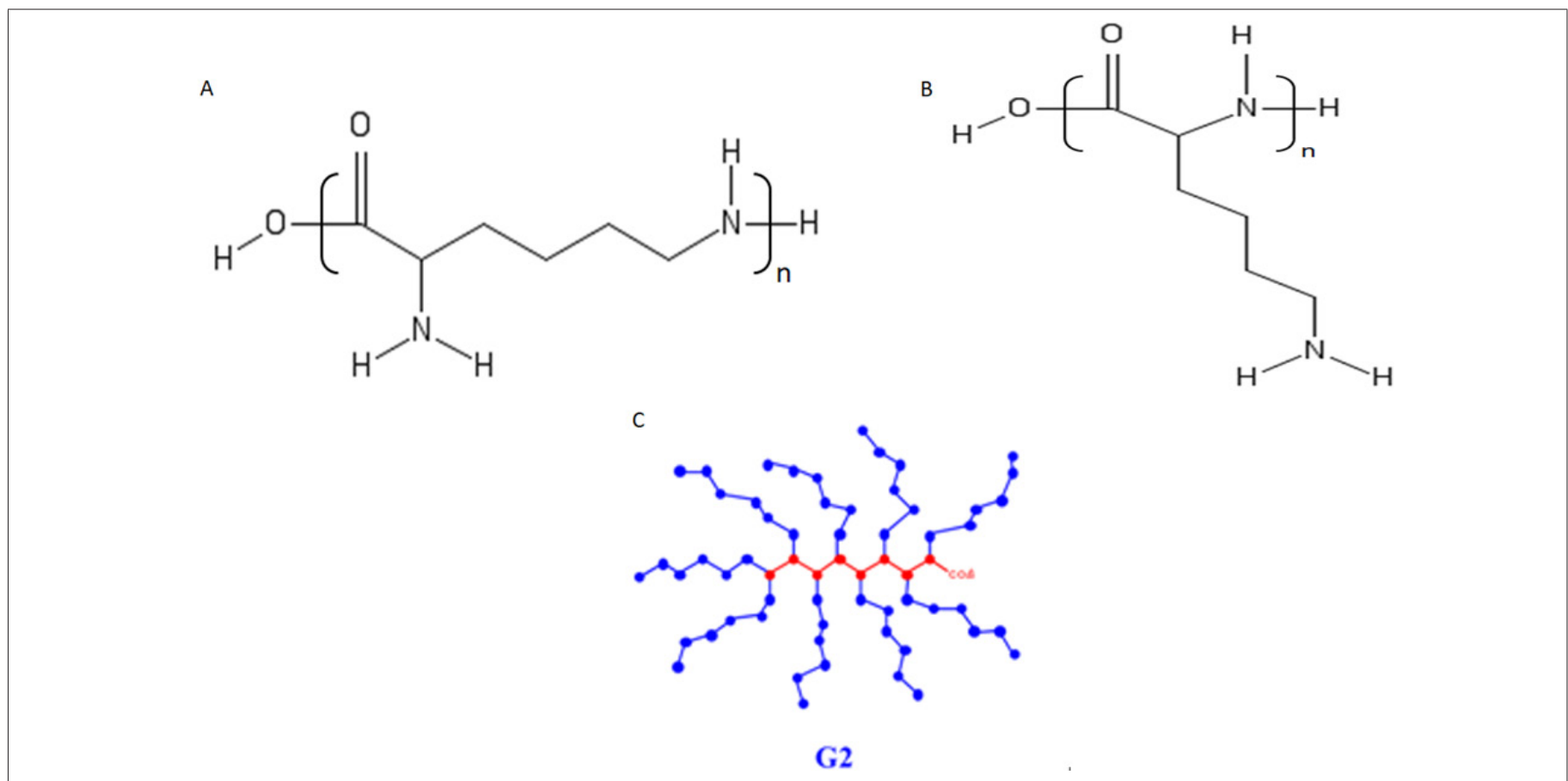

Figure T: Schemes of different structures of PLL: a a-PLL b, $\varepsilon-P L L ~ c, P L L$ dendrigraft G2 [25] is a combination of poly-L-lysines with an average of 48 Lys residues. 


\section{Cellular material preparation}

The iron foam was prepared from iron powder as reported in the literature [26]. Shortly, cellular iron structures were prepared by the impregnation of Polyurethane Foam (PUR), (Filtren ${ }^{\circledR}$ TM 25133 with the cell size $1060-1600 \mu m$, Euro foam TP spol. s ro, Brno, Czech Republic) with a suspension formed by $7 \mathrm{~g}$ of Carbonyl Iron Powder (CIP) provided by BASF, Ludwigshafen, Germany (type

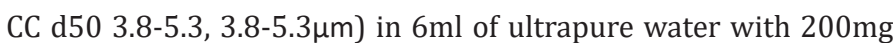
of gelatin. Gelatin (Sigma-Aldrich, St. Louis, MO 63103, USA) was dissolved in water at $60^{\circ} \mathrm{C}$. The composition of CIP was $99.5 \%$ $\mathrm{Fe}, 0.05 \% \mathrm{C}, 0.01 \% \mathrm{~N}$, and $0.18 \% \mathrm{O}$. The foam impregnation was carried out for $24 \mathrm{~h}$. Then the prepared structures were sintered in a tube furnace Aneta 1 (ANETA, Trenčianská Teplá, Slovak Republic) At first, the formed material was held at $450^{\circ} \mathrm{C}$ for $2 \mathrm{~h}$ in a nitrogen atmosphere. The final structure was obtained by sintering at $1120^{\circ} \mathrm{C}$ for $1 \mathrm{~h}$ in a reduction atmosphere $\left(90 \% \mathrm{~N}_{2}, 10 \% \mathrm{H}_{2}\right)$, resulting in the full removal of the polyurethane foam.

\section{Amino acid-based L-lysine layer preparation}

Amino acid-based layer was prepared by dipping prepared samples into $1 \mathrm{ml}$ of the coating solution for 360 minutes. In the case of L-lysine layers, solutions of $0.2,0.4,0.6$ and $0.8 \mathrm{~g} / \mathrm{l}$ were used. In the case of $\alpha$-PLL there were used solutions of 0.2 and $0.8 \mathrm{~g} / \mathrm{l}$. The $\mathrm{pH}$ of all coating solutions was set to be about 4 to keep all amino groups protonated by adding an $\mathrm{HCl}$ solution. After coating, samples were rinsed with water, dried in a stream of nitrogen, and stored in desiccators for further use.

\section{Electrochemical preparation of $\varepsilon$-poly-L-lysine layer}

Prior to modification, iron foams were sonicated in ethanol for 10 minutes. The electro polymerization of $\varepsilon$-poly-L-lysine was performed in a $1 \mathrm{X}$ PBS pH 9.0 containing $10 \mathrm{mM}$ L-lysine by cyclic voltammetry from -0.6 to $2.2 \mathrm{~V}$ for 5 cycles with a scan rate of $100 \mathrm{mV}$ s-1 using potentiostat Autolab 302N (Metrohm, Netherlands). Electro polymerization was performed in $50 \mathrm{ml}$ of solution. After electro polymerization samples were rinsed in purified water and dried in an oven at $50^{\circ} \mathrm{C}$ for 1 hour. Electrochemically induced polymerization reaction of $\alpha$-L-lysine, as it is presented in this study, leads to favorization of only $\varepsilon$ form of PLL which is biologically active and non-toxic. Very similar way of PLL preparation was presented in [27], where final $\varepsilon$ form is present and PLL structure was confirmed by Reflection-absorption infrared spectroscopy. There also exists another way to prepare $\varepsilon$ form PLL, but they are much more difficult [28].

\section{Corrosion measurements of $\varepsilon$-PLL modified carbonyl metallic foam}

Potentiodynamic polarization tests were performed using $\mathrm{Ag} /$ $\mathrm{AgCl} / \mathrm{KCl}$ (3mol L-1) as a reference electrode, a platinum plate as a counter electrode and the iron cellular structure, pure and PLL coated sample in the form of the foam as a working electrode. All measurements were conducted on multichannel potentiostatgalvanostat Autolab M204 (Metrohm, Herisau, Switzerland) at a $0.1 \mathrm{mV} \mathrm{s}-1$ scan rate. $1 \mathrm{X}$ PBS solution with $\mathrm{Ca}$ and $\mathrm{Mg}$ at a room temperature was used as a corrosive medium. Measurements of the Open Circuit Potential (OCP) were performed (with duration of 60 minutes) before corrosion tests under the conditions described above to reach the equilibrium state of the studied materials. All Measurement were run in triplicates. The most representative curves were chosen. Measured samples had thereabouts same active surface.

\section{Cell Culture}

Human dermal fibroblasts were cultured in DMEM medium with $1 \%$ Pen-Strep supplemented with $10 \%$ FBS in an incubator at $37^{\circ} \mathrm{C}$ with $5 \% \mathrm{CO}_{2}$. Cell seeding was performed in the same medium supplemented with $1 \%$ FBS. All coated samples were sterilized under UV light for 30 minutes then samples were flipped using sterile forceps and another side was UV light irradiated for the same time. After sterilization samples were transferred to the $2 \mathrm{ml}$ Eppendorf tube with 50000 cells in $1 \mathrm{ml}$ of medium and agitated on laboratory rocker softly do prevent cell settling for 4 hours. Then the samples with adhered fibroblasts were transferred to the 48 well plate with $0.8 \mathrm{ml}$ of the above-mentioned medium and stored in an incubator at before mentioned conditions.

\section{Viability tests and proliferation}

Viability tests were performed on a microplate reader FLUOstar OPTIMA FL BMG LABTECH, Germany. Before the test culturing medium was aspirated and samples were rinsed in PBS with Ca and Mg. After samples were rinsed $0.8 \mathrm{ml}$ of Deep Blue fluorescent assay was added to each sample and incubated at $37^{\circ} \mathrm{C}$ with $5 \% \mathrm{CO}_{2}$ for 2 hours. After incubation $100 \mu$ metabolized, Deep Blue assay was transferred to 96-well plate. The rest of the metabolized assay was aspirated, samples were rinsed, and fresh medium supplemented with $1 \%$ FBS was added and cultivation continued. Deep Blue assay was measured three times (after 24, 48 and 96 hours). There were 6 measurements performed on each sample.

\section{Cell fixation}

Cells were fixed in $2 \%$ glutaraldehyde in sodium phosphate buffer for 60 minutes at room temperature. After fixation, prior to the SEM observation samples were dried in ethanol solutions 50\%, $70 \%$ and $99 \%$ for 15 minutes each at room temperature. The rest of the alcohol solution was evaporated in a stream of nitrogen.

\section{SEM analyses}

SEM images were taken by Philips ESEM XL30, scanning electron microscope at a low vacuum to prevent biological structure damage. 


\section{General DFT Quantum ESPRESSO calculations of geometries}

The molecular models of PLL were well documented [29]. One model showed whole molecule of PLL with characteristic brushes of carbon chain. Hybrid functionals are already the de facto standard in quantum chemistry and are quickly gaining popularity in the condensed-matter physics and computational materials science communities. Hybrid functionals reduce the self-interaction error that plagues lower-rung exchange-correlation functionals, thus achieving more accurate and reliable predictive capabilities. This is of particular importance in the calculation of orbital energies, which are an essential ingredient in the treatment of band alignment and charge transfer in heterogeneous systems, as well as the input for higher-level electronic-structure calculations based on manybody perturbation theory. However, the widespread use of hybrid functionals is hampered by the often-prohibitive computational requirements of the exact-exchange (Fock) contribution, especially when working with a plane-wave basis set. The basic ingredient here is the action $\left(V^{\wedge} x \varphi i\right)(r)$ of the Fock operator $V^{\wedge} x$ onto a (single particle) electronic state $\varphi i$, requiring a sum over all occupied Kohn-Sham (KS) states $\{\psi j\}$.

For spin-unpolarized systems, one has: $\left(V^{\wedge} x \varphi i\right)(r)=-e 2 X j \psi j(r) Z d r 0 \psi * j(r 0) \varphi i(r 0)|r-r 0|$ (1) where-e is the charge of the electron. In the original algorithm [30] implemented in PWscf, self-consistency is achieved via a double loop: in the inner one the $\varphi$ 's entering the definition of the Fock operator in Equation (1) are kept fixed, while the outer one cycles until the Fock operator converges to within a given threshold. In the inner loop, the integrals appearing in Equation (1): $\operatorname{vij}(r)=Z d r 0 \rho i j(r 0)|r-r 0|, \rho i j(r)=\psi * i(r) \varphi j(r)$, (2) are computed by solving the Poisson equation in reciprocal space using fast Fourier Transforms (FFT). While feasible in relatively small cells, this unfavorable scaling with the system size makes calculations with hybrid functionals challenging if the unit cell contains more than a few dozen atoms. To enable exact-exchange calculations in the condensed phase, various ideas have been conceived and implemented in recent Quantum ESPRESSO versions 19

\section{Conclusion}

We present a study of both experimental and computational chemistry of carbonyl iron (110) surface modified with different structures of PLL. This modification enables adjustment of the surface hydrophobicity and different repulsion for fibroblast cells adhesion and growth to bone implant. Based on DFT Quantum ESPRESSO modelling the adhesion energies for every structure of the surfaces of carbonyl iron covered with $\alpha-/ \varepsilon$-PLL were calculated. The lowest adsorption energy and surface energy were calculated for $\alpha$-PLL with contact angle $45.7^{\circ}$ on the surface and highest adhesion energy (higher surface energy) for $\varepsilon$-PLL with value estimated $75.1^{\circ}$. These values predominate in interaction ranges from very hydrophilic (more repellent for cells) to a slightly hydrophobic (more attractive for fibroblast). Calculated results agreed with experimental results where adhesion of fibroblast cells on $\alpha$-PLL modified Fe (110) surface was investigated for the purpose of potential use as bone implants.

It was found out that the $\alpha$-PLL modified Fe (110) surface is more hydrophilic with fibroblast viability at borders 28000. $\varepsilon$-PLL caused Fe (110) surface was more hydrophobic and fibroblast viability increased slightly up to 35000 units. This work presents the structural modeling and subsequent DFT computational work in connection with experimental study for the purpose of the complex molecular interactions, especially in cases where experimental measurements are hindered. Mainly in the case when contact angle and hydrophobicity of surface is obstructed to find experimentally. The interaction of different fibroblast cells with the smart surfaces was demonstrated. In future work, we will study smart surfaces for different attraction of fibroblast at bone implant. Most interesting in the next research work will be studying the interaction with PLL dendrigrafts.

\section{Acknowledgement}

This research has been financially supported by APVV-160029 of the Slovak Research and Development Agency and VEGA 1/0095/21 of the Slovak Scientific Grant Agency. VS thanks Generalitat Valenciana (CIDEGENT/2018/036) and University Jaume I (B-2020-44) for funding.

\section{Conflict of Interest}

No conflict of interest.

\section{References}

1. CH Ho, E Odermatt, I Berndt, JC Tiller (2008) Ways of Selective Polycondensation of L-Lysine Towards Linear $\alpha$ - and $\varepsilon$-Poly-L-Lysine. J Polym Sci Part A Polym Chem 46(15): 5053-5063.

2. SK Tripathy, J Kumar, HS Nalwa, eds. (2002) Handbook of Polyelectrolytes and Their Applications, American Scientific Publishers. Stevenson Ranch CA.

3. G Decher (1997) Fuzzy nano assemblies: Toward layered polymeric multicomposites. Science 80(277): 1232-1237.

4. MF Villegas, L Garcia-Uriostegui, O Rodríguez, I Izquierdo-Barba, AJ Salinas, et al. (2017) Lysine-grafted MCM-41 silica as an antibacterial biomaterial. Bioengineering 4(4): 80 .

5. A Jindal, N Yadav, K Dhar, RG Moulick, J Bhattacharya (2019) Bifunctionalization of glass surfaces with poly-l-lysine conjugated silica particles and polyethylene glycol for selective cellular attachment and proliferation. J Mater Sci 54: 2501-2513.

6. JH Choi, SO Kim, E Linardy, EC Dreaden, VP Zhdanov (2015) Influence of $\mathrm{pH}$ and Surface Chemistry on Poly (1-lysine) Adsorption onto Solid Supports Investigated by Quartz Crystal Microbalance with Dissipation Monitoring. J Phys Chem B 119(33): 10554-10565. 
7. J Guo, Y Wei, D Zhou, P Cai, X Jing, et al. (2011) Chemosynthesis of poly( $\varepsilon$-lysine)-Analogous polymers by microwave-assisted click polymerization. Biomacromolecules 12(3): 737-746.

8. MA Fallah, C Stanglmair, C Pacholski, K Hauser, (2016) Devising SelfAssembled-Monolayers for Surface-Enhanced Infrared Spectroscopy of pH-Driven Poly-l-lysine Conformational Changes. Langmuir 32(29): 7356-7364.

9. L Hamadi, S Mansouri, K Oulmi, A Kareche (2018) The use of amino acids as corrosion inhibitors for metals: A review. Egypt J Pet 27(4): 11571165 .

10. S Zor, F Kandemirli, M Bingul (2009) Inhibition effects of methionine and tyrosine on corrosion of iron in $\mathrm{HCl}$ solution: Electrochemical, FTIR, and quantum-chemical study. Prot Met Phys Chem Surfaces 45(1): 46-53.

11. MA Amin, KF Khaled, Q Mohsen, HA Arida (2010) A study of the inhibition of iron corrosion in $\mathrm{HCl}$ solutions by some amino acids. Corros Sci 52(5):1684-1695

12. E Varoni, E Canciani, B Palazzo, V Varasano, P Chevallier, et al. (2015) Effect of poly-l-lysine coating on titanium osseointegration: From characterization to in vivo studies. J Oral Implantol 41(6): 626-631.

13. EM Harnett, J Alderman, T Wood (2007) The surface energy of various biomaterials coated with adhesion molecules used in cell culture. Colloids Surfaces B Biointerfaces 55(1): 90-97.

14. JE Raynor, JR Capadona, DM Collard, TA Petrie, AJ García (2009) Polymer brushes and self-assembled monolayers: Versatile platforms to control cell adhesion to biomaterials (Review). Biointerphases 4(2): 3-16.

15. P Giannozzi, O Andreussi, T Brumme, O Bunau, MB Nardelli, et al. (2017) Advanced capabilities for materials modelling with Quantum ESPRESSO. J Phys Condens Matter 29(46): 465901.

16. M Fronzi, MHN Assadi, DAH Hanaor (2019) Theoretical insights into the hydrophobicity of low index $\mathrm{CeO}_{2}$ surfaces. Appl Surf Sci 478: 68-74

17. M Scarsi, N Majeux, A Caflisch (1999) Hydrophobicity at the surface of proteins. Proteins 37(4): 565-575.

18. F Tielens, V Humblot, CM Pradier, (2008) Elucidation of the low coverage chiral adsorption assembly of l-lysine on $\mathrm{Cu}$ (110) surface: A theoretical study. Surf Sci 602: 1032-1039.

19. T Eralp, A Shavorskiy, G Held (2011) The adsorption geometry and chemical state of lysine on $\mathrm{Cu}\{110\}$. Surf Sci 605(3-4): 468-472.
20. D Zhang, Z Cheng, Y Liu (2019) Smart Wetting Control on Shape Memory Polymer Surfaces. Chem 25(16): 3979-3992.

21. H Zhang, J Jiao, H Jin (2019) Degradable poly-L-lysine-modified PLGA cell microcarriers with excellent antibacterial and osteogenic activity. Artif Cells Nanomedicine Biotechnol 47(1): 2391-2404.

22.F Likibi, B Jiang, B Li (2008) Biomimetic nanocoating promotes osteoblast cell adhesion on biomedical implants. J Mater Res 23: 3222 3228.

23. IL Shih, MH Shen, YT Van (2006) Microbial synthesis of poly( $\varepsilon$-lysine) and its various applications. Bioresour Technol 97: 1148-1159.

24. SC Shukla, A Singh, AK Pandey, A Mishra (2012) Review on production and medical applications of e\{open\}-polylysine. Biochem Eng J 65: 7081.

25. M Scarsi, N Majeux, A Caflisch (1999) Hydrophobicity at the surface of proteins. Proteins 37(4): 565-575.

26. N Sisavath, T Le Saux, L Leclercq, H Cottet (2014) Effect of Dendrimer Generation on the Interactions between Human Serum Albumin and Dendrigraft Polylysines. Langmuir 30(15): 4450-4457.

27. R Oriňaková, R Gorejová, J Macko, A Oriňak, M Kupková, et al. (2019) Evaluation of in vitro biocompatibility of open cell iron structures with PEG coating. Appl Surf Sci 475: 515-518.

28. L Laurinavičius, A Radzevič, I Ignatjev, G Niaura, K Vitkutè, et al (2019) Investigation of electrochemical polymerisation of L-lysine and application for immobilisation of functionalised graphene as platform for electrochemical sensing. Electrochim Act 299: 936-945.

29. Y Tao, X Chen, F Jia, S Wang, C Xiao, et al. (2015) New chemosynthetic route to linear $\varepsilon$-poly-lysine. Chem Sci 6(11): 6385-6391.

30.Y Boughoues, M Benamira, L Messaadia, N Ribouh (2020) Adsorption and corrosion inhibition performance of some environmentally friendly organic inhibitors for mild steel in $\mathrm{HCl}$ solution via experimental and theoretical study. Colloids Surfaces a Physicochem Eng Asp 593: 124610.

31.JH Choi, SO Kim, E Linardy, EC Dreaden, VP Zhdanov, et al. (2015) Influence of $\mathrm{pH}$ and Surface Chemistry on Poly (l-lysine) Adsorption onto Solid Supports Investigated by Quartz Crystal Microbalance with Dissipation Monitoring. J Phys Chem B 119(3): 10554-10565. 